\title{
1 Females of the cellar spider discriminate against previous mates
}

2

Laia Mestre, José D. Rodríguez-Teijeiro \& Cristina Tuni

Laia Mestre, corresponding author: Department of Animal Biology, University of Barcelona, Avinguda Diagonal 645, 08028 Barcelona, Spain and Department of Ecology, Box 7044, Current address: Department of Ecology, Swedish University of Agricultural Sciences, Box 7044, 75007 Uppsala, Sweden, laiamesar@gmail.com.

José D. Rodríguez-Teijeiro: Department of Animal Biology, University of Barcelona, Avinguda Diagonal 645, 08028 Barcelona, jrodriguez@ub.edu.

Cristina Tuni: Ludwig Maximilians University Munich, Department Biology II, Großhaderner Str. 2, 82152 Planegg-Martinsried, Germany, cristina.tuni@bio.lmu.de.

Running title: Female preference for novel mates

Word count (Abstract-Literature cited): 4947

Key-words: polyandry, mate choice, sexual conflict, Pholcus phalangioides, Araneidae 


\section{Abstract}

Mate choice for novel partners should evolve when re-mating with males of varying genetic quality provides females with fitness-enhancing benefits. We investigated sequential mate choice for same or novel mating partners in females of the cellar spider Pholcus phalangioides (Pholcidae) to understand what drives female re-mating in this system. Females are moderately polyandrous and show high reluctance to re-mating. Yet, double-mated females benefit from a higher oviposition probability compared to single-mated females. We exposed mated females to either their former (same male) or to a novel mating partner, and assessed mating success together with courtship and copulatory behaviours in both sexes. We found clear evidence for mate discrimination: females experienced three-fold higher re-mating probabilities with novel males, being more often aggressive towards former males and accepting novel males faster in the second than in the first mating trial. Preference for novel males suggests that re-mating is driven by benefits derived from multiple partners. The low re-mating rates and the strong last male sperm precedence in this system suggest that mating with novel partners that represent alternative genotypes may be a means for selecting against a former mate of lower quality. 


\section{Introduction}

Litters, clutches and broods are commonly sired by more than one male (Birkhead \& Møller 1998; Taylor et al. 2014) suggesting that, despite the substantial costs incurred from re-mating (Knell \& Webberley 2004; Arnqvist \& Rowe 2005), polyandry (female multiple mating) is a pervasive mating strategy. Addressing female interests in the study of mating system evolution provides insight into its adaptive explanations (Arnqvist \& Nilsson 2000; Jennions \& Petrie 2000; Hosken \& Stockley 2003). By mating with multiple males females may enhance the chances of siring offspring of higher genetic quality or with optimal genetic compatibility (genetic benefits models) (Newcomer et al. 1999; Tregenza \& Wedell 2002). Females may also derive fecundity benefits by receiving male-derived resources as food, parental care or sperm (material benefits models) (Arnqvist \& Nilsson 2000). Polyandry may nevertheless also be the non-adaptive outcome of enforced matings with males that are able to overcome female reluctance to mate (sexual conflict models) (Holland \& Rice 1998; Arnqvist \& Rowe 2005).

Investigating mate choice for novel or previous mates may provide a useful tool to understand the underlying adaptive explanations for female re-mating. If females receive material fitness-enhancing benefits, for instance sperm supplies, selection should promote indiscriminate repeated matings regardless of whether they occur with the same or different mating partners (Ridley 1988; South \& Lewis 2011). On the contrary, females can only assure genetic benefits by varying the genetic quality of their mates to allow post-mating selection for best or most compatible genotypes through sperm competition or female fertilization biases (Jennions \& Petrie 2000; Simmons 2001; Bretman et al. 2009). In the latter case females are expected to avoid re-matings with previous mates. Indeed, in hamsters (Lisk \& Baron 1982), guppies (Hughes et al. 1999; Eakley \& Houde 2004), hide beetles (Archer \& Elgar 1999), crickets (Bateman 1998; Ivy et al. 2005), dung flies (Hosken et al. 2003) and pseudoscorpions 
(Zeh et al. 1998), polyandrous females are known to mate preferentially with novel males when given the opportunity.

Females can acquire better or more compatible genes for their offspring without necessarily enhancing post-mating selection mechanisms. In systems where last males to mate experience highest fertilization success (hereafter called last male sperm precedence), as some insects and arachnids (Birkhead \& Hunter 1990; Elgar 1998), female mate choice for novel mating partners will inevitably select against previous mates. In such systems, females may gain benefits for their offspring by re-mating exclusively when they encounter a male of superior quality compared to their previous mate, polyandry being used as a strategy to compensate for lower quality inseminations.

In this study we investigated female sequential mate choice for same or novel mating partners in the cellar spider Pholcus phalangioides (Pholcidae), a cosmopolitan web-building spider whose reproductive behaviour has been extensively studied (Uhl 1998; Schäfer \& Uhl 2002; Schaefer \& Uhl 2003; Schäfer \& Uhl 2005; Uhl et al. 2005). Females are moderately polyandrous ( $83.3 \%$ of wild-caught broods are sired by more than one male, with an average of 2.5 sires, Schultz and Uhl unpublished) even though one mating is sufficient to produce fertile egg sacs throughout the female's lifetime (Uhl 1993). Laboratory studies show that females do not readily re-mate: female acceptance of first matings is close to $100 \%$ but drops to $68-82 \%$ in second matings (Schäfer \& Uhl 2002; Schäfer \& Uhl 2005; Schäfer et al. 2008). Males cannot enforce copulations, because females need to position themselves horizontally and expose their genital opening, so that males can secure their mouthparts to a sclerotized hook of the female's genital plate and introduce both of their pedipalps (Uhl et al. 1995). The reproductive interests over re-mating between the sexes appear to be in conflict. Due to last male sperm precedence, 
second males to mate fertilizing $88-89 \%$ of the offspring (Schäfer \& Uhl 2002; Schäfer et al. 2008), so it is in the males best interest to mate with already mated females.

Despite female reluctance to accept second mates, re-mating is known to be beneficial for female fitness, double-mated females experiencing higher oviposition probabilities compared to singlemated females (Uhl et al. 2005). What remains unclear is whether re-mating triggers oviposition through the reception of more sperm and/or stimulants in the ejaculate (direct benefit models), which could also be achieved by mating repeatedly with the same male, or through the effect of multiple ejaculates (genetic benefit models) (Tregenza \& Wedell 1998).

We use female choice for novel or former mating partners to shed light on the adaptive explanation for polyandry in this system, as mate choice for male novelty should evolve exclusively when fitness benefits derive from multiple mates. We exposed mated females sequentially to either the same male as in their first mating (same male treatment, SM) or to a novel male, which the female had never encountered (novel male treatment, NM) and scored differences in female pre-copulatory (aggressive interactions, acceptance of the mate, and latency to copulation) and copulatory behaviours (copulation duration) which were used as proxies for female mate choice. To control for differences in male mating effort towards novel or previous females we also scored male pre-copulatory (latency to courtship, coupling attempts) and copulatory (pedipalp movements) behaviours. If re-mating is beneficial due to the reception of multiple partners we expect females to mate preferentially with novel mates, whereas if remating is necessary to obtain sufficient sperm or sufficient oviposition stimulants females should re-mate regardless of male novelty.

\section{Methods}

\section{Collection, rearing and body measures}


Immature spiders during their penultimate (4th and 5th) sub adult stages were collected from several buildings in the city of Barcelona (Spain) and brought to the laboratory during spring 2008. Individuals were reared individually in (length, width, height) $16 \times 9 \times 9 \mathrm{~cm}$ transparent containers and were fed five Drosophila melanogaster twice a week and two crickets Acheta domestica once a week. Upon reaching adulthood, spiders were sexed and the tibia-patella length of the first right leg was measured and used as a proxy for body size (Schäfer et al. 2008). Each individual was anaesthetized with $\mathrm{CO}_{2}$, placed onto a squared-millimeter paper with the legs parallel to the surface and a photograph was taken with a Canon EOS 350D camera. Images were then processed with SigmaScan Pro 5.0.0 software to obtain the body measurements. All individuals (and their offspring) were released after the end of the study, in September 2008.

\section{Experimental design}

Virgin females were randomly assigned to either of the two treatments, "same male" (SM, $\mathrm{N}=38$ ) or "novel male" (NM, N=37). All females were first mated with a virgin male and were subsequently presented with a second male in order to re-mate. SM females were given the same male as in their first mating, whereas NM females a novel male which had previously mated to a virgin female of the same treatment. Hence, both NM and SM males, in the second mating, had the same mating status (mated once with a virgin female) (Tregenza \& Wedell 1998).

Since body size differences within a mating pair are known to affect copulatory behaviours in this species (Schäfer et al. 2008), males were assigned to females with matching patella-tibia length ( $\pm 0.3 \mathrm{~mm}$, see (Schäfer \& Uhl 2002)). There were no significant differences in tibiapatella lengths $(\mathrm{mm})$ between treatments in either females (mean $\pm \mathrm{SE})(\mathrm{SM}=11.70 \pm 0.27$; $\mathrm{NM}$ $\left.=12.15 \pm 0.17 ; \mathrm{F}_{1,73}=1.99, \mathrm{p}=0.16\right)$ or males $\left(\mathrm{SM}=11.68 \pm 0.27 ; \mathrm{NM}=12.14 \pm 0.17 ; \mathrm{F}_{1,73}=\right.$ $1.95, \mathrm{p}=0.17)$ 
All spiders were mated within 45 days after their final moult to adulthood to prevent age affecting copulatory behaviour, as it is known that number of PPM decreases with male age (Schäfer \& Uhl 2002). There were no significant differences in age (number of days) between treatments in either females $\left(\mathrm{SM}=33.82 \pm 1.49 ; \mathrm{NM}=32.60 \pm 1.41 ; \mathrm{F}_{1,73}=0.36, \mathrm{p}=0.55\right)$ or males $\left(\mathrm{SM}=29.45 \pm 1.78 ; \mathrm{NM}=31.70 \pm 1.53 ; \mathrm{F}_{1,73}=0.92, \mathrm{p}=0.34\right)$. Individuals of a mating pair were of the same age (females $=33.21 \pm 1.02$; males $=30.56 \pm 1.18$; paired t-test: $t_{74}=1.66$, $\mathrm{p}=0.1)$. Females were transferred to a new container $(14.5 \times 9 \times 6.5 \mathrm{~cm}) 12$ hours before the scheduled mating to give them time to spin a web and acclimatize to the new environment. Mating boxes were only used a single time to prevent intermixing of chemical cues between mating trials. Thus, 150 mating boxes were used in total. Females of each treatment were given a second male between 3 and 5 hours after the first mating because female re-mating probability drops steeply after 6 hours (Schäfer \& Uhl 2005). This also allows males to perform recharge their pedipalps with sperm after the first copulation. A sub-sample of males $(N=20)$ was constantly monitored through direct observations and/or video recording to document male recharge of pedipalps. Sperm induction in $P$. phalangioides has been described as males hanging upside down in their web and taking the seminal drop with the third pair of legs; the drop is placed between the chelicerae and then the male dips the pedipalps alternatively into the drop to charge them (Gerhardt (1927) in Huber (1998)).

\section{Behavioural observations}

Courtship is almost always initiated by the male. Males can begin courtship with different behaviours, such as performing abdominal vibrations, tapping and jerking the female's web, and tapping the female's legs using his first pair of legs (Bartos 1998). Females do not move during courtship, though they can produce web vibrations. We considered courtship to start upon the first contact of a male with the female because the other behaviours are difficult to detect and can be missing from the courtship sequence, whereas tapping of the female's legs always happens 
(Bartos 1998). In both first and second mating trials we scored the following pre-copulatory variables: latency to courtship, defined as the time from the start of the experiment to the first physical contact between a male and a female; occurrence of aggressive interactions (yes/no), defined as females rejecting males by pushing the male away with front leg-movements and/or chasing the male; latency to copulation, defined as the time from courtship to copulation; and number of coupling attempts, since males typically perform several attempts before introducing their pedipalps into the genital cavity and start copulation. While latency to courtship and coupling attempts are behaviours under male control, we use latency to copulation and aggressive interactions as proxies for female willingness to mate. A mating trial began when a male was introduced into the female box. The pair was then given 90 minutes to start copulation (Schäfer \& Uhl 2002). If the pair did not mate within this timeframe, the male was removed from the box and the mating trial scored as failed. The copulatory variables scored were number of pedipalp movements (PPM) and copulation duration. PPMs are rhythmic movements that males make during copulation with both inserted pedipalps and that correlate positively with the amount of sperm transferred (Uhl unpublished) and paternity success (Schäfer \& Uhl 2002). All successful copulations were observed until male pedipalps became detached from the female genitalia.

For those males monitored during sperm induction we recorded: latency to sperm induction, defined as the time elapsed from the first copulation until start of sperm uptake; induction duration, defined as the total time spent recharging its pedipalps; and number of sperm uptake movements. We considered induction to start when the third pair of legs was bent to contact with the genital pore and we considered it to end when the male un-bended the legs. All behavioural observations were done by LM.

\section{Statistical analyses}


We used generalized linear models (GLM) with Gamma (GLM-g) distribution for continuous variables (latency to courtship, latency to copulation, copulation duration), Poisson (GLM-p) distribution (log-link function, corrected for overdispersion) for count variables (number of coupling attempts, number of PPM) and Binomial (GLM-b) distribution (logit-link function, corrected for overdispersion) for dichotomous variables (occurrence of aggressive interactions), and ran the models on the effect of treatment (SM or NM) for the first mating to ensure that behaviours scored did not differ. Treatment effects (SM, NM) on female re-mating decisions (accept/reject second copulation) and occurrence of aggressive interactions (yes/no) were analysed with GLM using the Binomial (GLM-b) distribution (logit-link function, corrected for overdispersion). In all these models that tested treatment effects we included "body size difference" (absolute value of the body size difference between the members of the mating pair) and "age" (age of the oldest individual of the mating pair) and their interactions with treatment to control for the potentially confounding effects of these variables (see "Experimental design" above). Finally we employed generalized linear mixed models (GLMM) to account for female individual autocorrelation, with the above-mentioned error distributions, and ran the model on the effect of mating trial number (first and second mating) on mating behaviours within each treatment (SM and NM), including "body size difference" and "age" as covariates. We always started with full models and used manual stepwise backward selection to discard nonsignificant effects, which led to final models only including the effects of treatment or of mating trial number, or to no effects at all. We conducted all analyses using $\mathrm{R}$ version 3.1.0 (R Development Core Team 2014), with the package MASS for mixed models (Venables \& Ripley 2002). Results are shown as the mean $\pm \mathrm{SE}$.

\section{Results}

\section{First mating trials}


All virgin females mated with the first male. None of the behavioural variables differed between SM and NM treatments (GLM-b, occurrence of aggressive interactions $=0.07 \pm 0.03$; GLM-g, latency to courtship $=2.94 \pm 0.74$ minutes, latency to copulation $=19.48 \pm 2.42$, copulation duration $=80.22 \pm 3.81$ minutes; GLM-p, number of coupling attempts $=15.60 \pm 2.96$, number of $\mathrm{PPM}=185.00 \pm 6.98$, all $\mathrm{p}>0.21)$.

\section{Second mating trials}

Re-mating probability in NM females was more than three times as high as in the SM treatment: only 3 out of 38 females of the SM treatment re-mated, compared to 10 out of 27 females of the NM treatment (GLM-b, $\chi_{1,73}^{2}=64.17, p=0.025$; Fig. 1), and the probability of a female being aggressive towards the male was more than four times as high in the SM as in the NM treatment $\left(\mathrm{SM}=0.75 \pm 0.074, \mathrm{NM}=0.16 \pm 0.061 ; \chi_{1,71}^{2}=73.29, \mathrm{p}<0.0001\right)$

$$
\text { There was no difference between treatments in male latency to courtship (GLM-g, } F_{1,70}=
$$
2.57, $\mathrm{P}=0.11$; Fig. 2a). However, comparisons of first and second mating trials showed that males initiated courtship sooner when they were presented the same female a second time, as latency to courtship was lower in second than in first matings in the SM treatment $\left(\mathrm{F}_{1,34}=8.41\right.$, $\mathrm{p}=0.0065)$ but not in NM males $\left(\mathrm{F}_{1,36}=0.14, \mathrm{p}=0.71 ;\right.$ Fig. $\left.2 \mathbf{b}\right)$.

$$
\text { and second mating trials: mated females accepted males faster but copulated for a much shorter }
$$
time, whereas mated males performed a higher number of coupling attempts but did less PPMs than in the first mating (Table 1). All second copulations in both SM and NM treatments were short [<47 PPM, sensu (Schäfer et al. 2008)], except for one NM copulation with 189 PPM that lasted for 79.78 minutes, which was excluded from data analysis when comparing first and second matings. Due to the low number of SM females that accepted to re-mate $(\mathrm{N}=3)$, number 
of coupling attempts and copulatory variables between first and second matings in this treatment group are not compared.

\section{Sperm induction}

Males initiated post-mating sperm induction one hour and a half after the first copulation (induction latency: $88.72 \pm 3.94$ minutes, $N=20$ ). Sperm induction lasted on average $5.13 \pm$

0.30 minutes $(\mathrm{N}=17)$ and included $7.17 \pm 0.83$ sperm uptake movements $(\mathrm{N}=12)$.

\section{Discussion}

Our study revealed that Pholcus phalangioides females discriminate against previous mates

when given the opportunity to re-mate. Females exposed to novel males exhibit three-fold higher re-mating probabilities than those encountering former mating partners. In accordance to such finding, females were more aggressive towards former mates, rejecting and chasing former males in higher proportions compared to males that had not been encountered previously. Novel males presented in second mating trials were also accepted sooner than virgin novel males presented in first mating trials. Altogether our data show that females prefer re-mating with novel males.

Although we could not test for reproductive success differences between treatments due to the very low numbers of females accepting a second mate, female mate choice patterns may indirectly shed light on the adaptive explanations for re-mating in this system. Preference for novel males implies that fitness benefits of re-mating arise from the ability to select for multiple different partners. Due to $P$. phalangioides low re-mating rates (27\% in the NM treatment) and last male sperm precedence — second males to mate are known to mechanically remove sperm (i.e. eject or translocate) from previous males via pedipalp movements (Schäfer \& Uhl 2002), 
that polyandry is a means to promote post-mating processes selecting for best or most compatible genes (Jennions \& Petrie 2000; Simmons 2001). Mating with a novel male may prove beneficial if the male's quality exceeds the quality of a previous mate, mate choice predicting fertilization outcome. Hence, previously reported higher oviposition probabilities of double- compared to single-mated $P$. phalangioides females (Uhl et al. 2005) may have been triggered by mating with a novel mating partner and not by the number of matings per se. The novel mate may overcome low quality inseminations from males unsuccessful in triggering oviposition due to infertility, failure of sperm transfer or due to lack of oviposition stimulants in their ejaculates (Jennions \& Petrie 2000). Insect males are known to transfer substances in their seminal fluids which are able to stimulate ovulation by enhancing female production of egglaying hormones (Lange 1984; Stanley 2006), or by directly interfering with females neuronal pathways (Rubinstein \& Wolfner 2013). In addition, since seminal fluids are complex mixtures of molecules exhibiting large between-male variation in their composition (Poiani 2006) it may be the synergistic effect of multiple stimulants inside different ejaculates to ultimately trigger oviposition (Eady et al. 2000). Although seminal fluid composition has not yet been characterized in spiders, similar processes are likely to occur, and positive effects of multiple ejaculates on oviposition probabilities have been documented in this group (Tuni et al. 2013).

$$
\text { much lower than that reported in previous studies }(>68 \%) \text { despite similar experimental }
$$
protocols (Schäfer \& Uhl 2002, 2005; Uhl et al. 2005; Schäfer et al. 2008). The only difference is that previous studies used virgin males in second mating trials, whereas we used once-mated males. This suggests that females may prefer mating with virgin than with non-virgin males, a strategy possibly adopted to avoid males with declining reproductive output following consecutive matings (i.e. sperm depletion) (South and Lewis 2011), or reduce the risk of sexually transmitted disease infection (Knell and Webberley 2004). 
Sexual interest in males is known to decline after repeated matings with the same female,

while it restores when experiencing novel mating partners (Wilson et al. 1963; Dewsbury 1981).

Such phenomenon, known as 'Coolidge effect', is driven by prudent allocation of costly male resources (sperm and seminal fluids) based on female quality (Wedell et al. 2002). Low sample sizes prevented us from comparing number of pedipalp movements between treatments which, as indicators for sperm allocation (Schäfer \& Uhl 2002), would allow full detection of differences in male investment. Nevertheless, by comparing the latency of male initiation of courtship between treatments we can exclude that the higher re-mating probability with novel partners is due to greater male attraction to novel females (Gershman \& Sakaluk 2009; Tuni \& Bilde 2010; Werminghausen et al. 2013) (but see (Steiger et al. 2008)). On the contrary, males started courting former mates sooner compared to novel ones. Thus, males may be able to recognize previously mated females, for example via pheromones (Gaskett 2007), and adjust their behaviour accordingly. This could be interpreted as a strategy for males to reduce the costs of mating when exposed to a new individual (i.e. disease or parasite transmission) (Daly 1978; Kokko et al. 2002)

We conclude that females of the cellar spider discriminate against previous mates. The fitness benefits to choosy females should select for mate recognition, though the mechanisms through which females discriminate males remain unexplored. Complex vibratory and/or chemical signals may operate in concert while males approach females on their webs allowing females to assess male identity (Huber 2005).

\section{Acknowledgements}

We are grateful to Guillem Pascual and Carles Ribera for help with spider collection and to Helena Bassas for help with spider keeping. We also thank Empar Mondéjar for fruit fly supply 
and to Marta Pascual for granting us access to his lab to anesthetize the spiders. The Catalan

"Direcció General de la Recerca" (2005-SGR-00090) gave financial support.

\section{Literature cited}

Archer, M. S. \& Elgar, M. A. 1999: Female preference for multiple partners: sperm competition in the hide beetle, Dermestes maculatus (De Geer). Anim Behav 58, 669-675.

Arnqvist, G. \& Nilsson, T. 2000: The evolution of polyandry: multiple mating and female fitness in insects. Anim Behav 60, 145-164.

Arnqvist, G. \& Rowe, L. 2005: Sexual Conflict: Sexual Conflict. Princeton University Press, Princeton, New Jersey.

Bartos, M. 1998: Quantitative analyses of male courtship behaviour in Pholcus phalangioides (Fuesslin, 1775) (Araneae, Pholcidae). In: Proceedings of the 17th European Colloquium of Arachnology.

Bateman, P. W. 1998: Mate preference for novel partners in the cricket Gryllus bimaculatus. Ecol Entomol 23, 473-475.

Birkhead, T. \& Hunter, F. 1990: Mechanisms of sperm competition. Trends Ecol Evol 5, 48-52.

Birkhead, T. R. \& Møller, A. P. 1998: Sperm competition and sexual selection. Academic Press.

Bretman, A., Newcombe, D. \& Tregenza, T. 2009: Promiscuous females avoid inbreeding by controlling sperm storage. Mol Ecol 18, 3340-3345.

Daly, M. 1978: The cost of mating. Am Nat, 771-774.

Dewsbury, D. A. 1981: Effects of novelty of copulatory behavior: The Coolidge effect and related phenomena. Psychol Bull 89, 464.

Eady, P. E., Wilson, N. \& Jackson, M. 2000: Copulating with multiple mates enhances female fecundity but not egg-to-adult survival in the bruchid beetle Callosobruchus maculatus. Evolution 54, 2161-2165. 
Eakley, A. L. \& Houde, A. E. 2004: Possible role of female discrimination against 'redundant'males in the evolution of colour pattern polymorphism in guppies. Proc Roy Soc Lond B Bio 271, S299-S301.

Elgar, M. 1998: Sperm competition and sexual selection in spiders and other arachnids. In: Sperm Competition and Sexual Selection, (Birkhead, T. R. \& Møller, A. P., eds). Academic Press, California, pp. 307-339.

Gaskett, A. C. 2007: Spider sex pheromones: emission, reception, structures, and functions. Biol Rev 82, 27-48.

Gershman, S. N. \& Sakaluk, S. K. 2009: No Coolidge effect in decorated crickets. Ethology 115, 774-780.

Holland, B. \& Rice, W. R. 1998: Perspective: chase-away sexual selection: antagonistic seduction versus resistance. Evolution, 1-7.

Hosken, D., Martin, O., Born, J. \& Huber, F. 2003: Sexual conflict in Sepsis cynipsea: female reluctance, fertility and mate choice. J Evol Biol 16, 485-490.

Hosken, D. \& Stockley, P. 2003: Benefits of polyandry: a life history perspective. In: Evolutionary Biology, (MacIntyre, R. J. \& Clegg, M. T., eds). Springer, New York, pp. 173-194.

Huber, B. A. 1998: Spider reproductive behaviour: a review of Gerhardt's work from 1911-1933, with implications for sexual selection. Bull Brit Arachnol Soc 11, 81-91.

Huber, B. A. 2005: Sexual selection research on spiders: progress and biases. Biol Rev 80, 363385.

Hughes, K. A., Du, L., Rodd, F. H. \& Reznick, D. N. 1999: Familiarity leads to female mate preference for novel males in the guppy, Poecilia reticulata. Anim Behav 58, 907-916.

Ivy, T. M., Weddle, C. B. \& Sakaluk, S. K. 2005: Females use self-referent cues to avoid mating with previous mates. Proc Roy Soc B: Biol Sci 272, 2475-2478. 
Jennions, M. D. \& Petrie, M. 2000: Why do females mate multiply? A review of the genetic benefits. Biol Rev 75, 21-64.

Knell, R. J. \& Webberley, K. M. 2004: Sexually transmitted diseases of insects: distribution, evolution, ecology and host behaviour. Biol Rev 79, 557-581.

Kokko, H., Ranta, E., Ruxton, G. \& Lundberg, P. 2002: Sexually transmitted disease and the evolution of mating systems. Evolution 56, 1091-1100.

Lange, A. B. 1984: The transfer of prostaglandin-synthesizing activity during mating in Locusta migratoria. Insect Biochem 14, 551-556.

Lisk, R. D. \& Baron, G. 1982: Female regulation of mating location and acceptance of new mating partners following mating to sexual satiety: the Coolidge effect demonstrated in the female golden hamster. Behav Neurol Biol 36, 416-421.

Newcomer, S. D., Zeh, J. A. \& Zeh, D. W. 1999: Genetic benefits enhance the reproductive success of polyandrous females. P Proc Natl Acad Sci USA 96, 10236-10241.

Poiani, A. 2006: Complexity of seminal fluid: a review. Behav Ecol Sociobiol 60, 289-310.

R Development Core Team. 2014: R: A language and environment for statistical computing. R Foundation for Statistical Computing, Vienna, Austria. URL http://www.R-project.org/.

Ridley, M. 1988: Mating frequency and fecundity in insects. Biol Rev 63, 509-549.

Rubinstein, C. D. \& Wolfner, M. F. 2013: Drosophila seminal protein ovulin mediates ovulation through female octopamine neuronal signaling. Proc Natl Acad Sci USA 110, 1742017425.

Schaefer, D. \& Uhl, G. 2003: Male Competition over Access to Females in a Spider with Last-Male Sperm Precedence. Ethology 109, 385-400.

Schäfer, M. A., Misof, B. \& Uhl, G. 2008: Effects of body size of both sexes and female mating history on male mating behaviour and paternity success in a spider. Anim Behav 76, 7586. 
Schäfer, M. A. \& Uhl, G. 2002: Determinants of paternity success in the spider Pholcus phalangioides (Pholcidae: Araneae): the role of male and female mating behaviour. Behav Ecol Sociobiol 51, 368-377.

-. 2005: Sequential mate encounters: female but not male body size influences female remating behavior. Behav Ecol 16, 461-466.

Simmons, L. W. 2001: Sperm Competition and its Evolutionary Consequences in the Insects. Princeton University Press, Princeton, New Jersey.

South, A. \& Lewis, S. M. 2011: The influence of male ejaculate quantity on female fitness: a meta-analysis. Biol Rev 86, 299-309.

Stanley, D. 2006: Prostaglandins and other eicosanoids in insects: biological significance. Annu Rev Entomol 51, 25-44.

Steiger, S., Franz, R., Eggert, A.-K. \& Müller, J. K. 2008: The Coolidge effect, individual recognition and selection for distinctive cuticular signatures in a burying beetle. Proc Roy Soc B: Biol Sci 275, 1831-1838.

Taylor, M. L., Price, T. A. \& Wedell, N. 2014: Polyandry in nature: a global analysis. Trends Ecol Evol. DOI: 10.1016/j.tree.2014.04.005

Tregenza, T. \& Wedell, N. 1998: Benefits of multiple mates in the cricket Gryllus bimaculatus. Evolution, 52, 1726-1730.

-. 2002: Polyandrous females avoid costs of inbreeding. Nature 415, 71-73.

Tuni, C., Albo, M. \& Bilde, T. 2013: Polyandrous females acquire indirect benefits in a nuptial feeding species. J Evol Biol 26, 1307-1316.

Tuni, C. \& Bilde, T. 2010: No preference for novel mating partners in the polyandrous nuptialfeeding spider Pisaura mirabilis (Araneae: Pisauridae). Anim Behav 80, 435-442.

Uhl, G. 1993: Sperm storage and repeated egg production in female Pholcus phalangioides Fuesslin (Araneae). Bull Soc Neuchatel Sci Nat 116, 245-252. 
Uhl, G. 1994: Genital morphology and sperm storage in Pholcus phalangioides (Fuesslin, 1775) (Pholcidae; Araneae). Acta Zool 75, 1-12.

-. 1998: Mating behaviour in the cellar spider, Pholcus phalangioides, indicates sperm mixing. Anim Behav 56, 1155-1159.

Uhl, G., Huber, B. \& Rose, W. 1995: Male pedipalp morphology and copulatory mechanism in Pholcus phalangioides (Fuesslin, 1775) (Araneae, Pholcidae). Bull Brit Arachnol Soc 10, 1-9.

Uhl, G., Schmitt, S. \& Schäfer, M. A. 2005: Fitness benefits of multiple mating versus female mate choice in the cellar spider (Pholcus phalangioides). Behav Ecol Sociobiol 59, 6976.

Venables, W. N. \& Ripley, B. D. 2002: Modern Applied Statistics with S. Springer, New York.

Wedell, N., Gage, M. J. \& Parker, G. A. 2002: Sperm competition, male prudence and spermlimited females. Trends Ecol Evol 17, 313-320.

Werminghausen, J., Lange, R. \& Anthes, N. 2013: Seeking a Sex-Specific Coolidge Effect in a Simultaneous Hermaphrodite. Ethology 119, 541-551.

Wilson, J. R., Kuehn, R. E. \& Beach, F. A. 1963: Modification in the sexual behavior of male rats produced by changing the stimulus female. J Comp Physiol Psych 56, 636.

Zeh, J. A., Newcomer, S. D. \& Zeh, D. W. 1998: Polyandrous females discriminate against previous mates. Proc Natl Acad Sci USA 95, 13732-13736. 


\section{Figure Legends}

446

$447 \quad$ Fig. 1.

448 Female re-mating probability when exposed to same (SM) or novel (NM) male. Results are 449 shown as the mean $\pm \mathrm{SE}$.

450

$451 \quad$ Fig. 2.

452 Male latency to courtship during (a) same (SM) and novel (NM) male treatment and (b) during 453 first and second mating trials in the SM treatment. Results are shown as the mean \pm SE. 
Table 1.

455 Comparison of pre-copulatory and copulatory behaviours between first and second mating trials 456 with novel males (NM treatment).

457

\begin{tabular}{lcccccc}
\hline & \multicolumn{1}{l}{ First mating } & Second mating & N & GLMM \\
\hline Variable & mean & SE & mean & SE & & \\
\hline Courtship latency (min) & 2.57 & 1.19 & 2.12 & 0.75 & 37 & $\mathrm{~F}_{1,36}=0.14, \mathrm{p}=0.71$ \\
Copulation latency (min) & 17.36 & 5.60 & 6.61 & 1.53 & 9 & $\mathrm{~F}_{1,8}=6.49, \mathrm{p}=0.034$ \\
Number of coupling attempts & 10.33 & 1.89 & 28.67 & 18.77 & 9 & $\mathrm{~F}_{1,8}=6.78, \mathrm{p}=0.032$ \\
Copulation duration (min) & 93.12 & 10.42 & 1.30 & 0.63 & 9 & $\mathrm{~F}_{1,8}=7.97, \mathrm{p}=0.022$ \\
& & & & & & \\
Number of PPM & 190.22 & 12.92 & 10.33 & 4.62 & 9 & $\mathrm{~F}_{1,8}=56.47, \mathrm{p}=0.0001$ \\
\hline
\end{tabular}

458

459

460 
Fig. 1.

462 Female re-mating probability when exposed to same (SM) or novel (NM) male. Results are 463 shown as the mean $\pm \mathrm{SE}$.

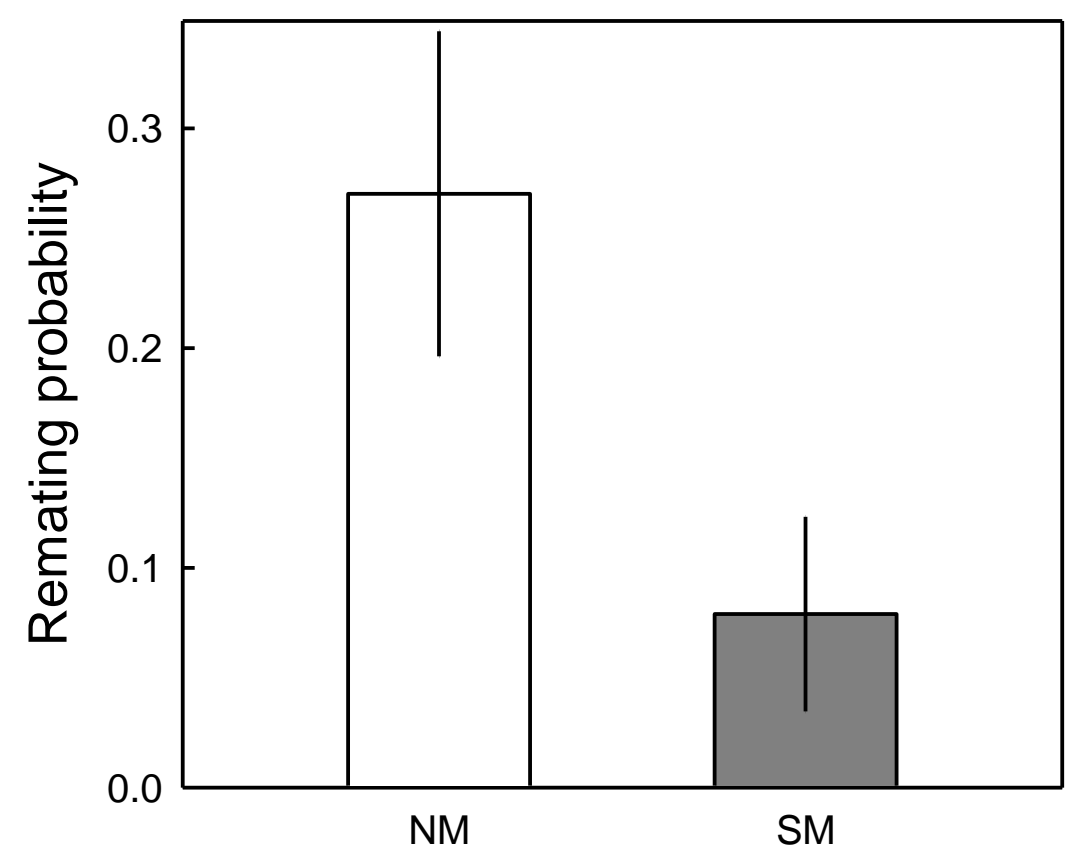

464

465 
Fig. 2.

467 Male latency to courtship during (a) same (SM) and novel (NM) male treatment and (b) during 468 first and second mating trials within each treatment. Results are shown as the mean \pm SE.
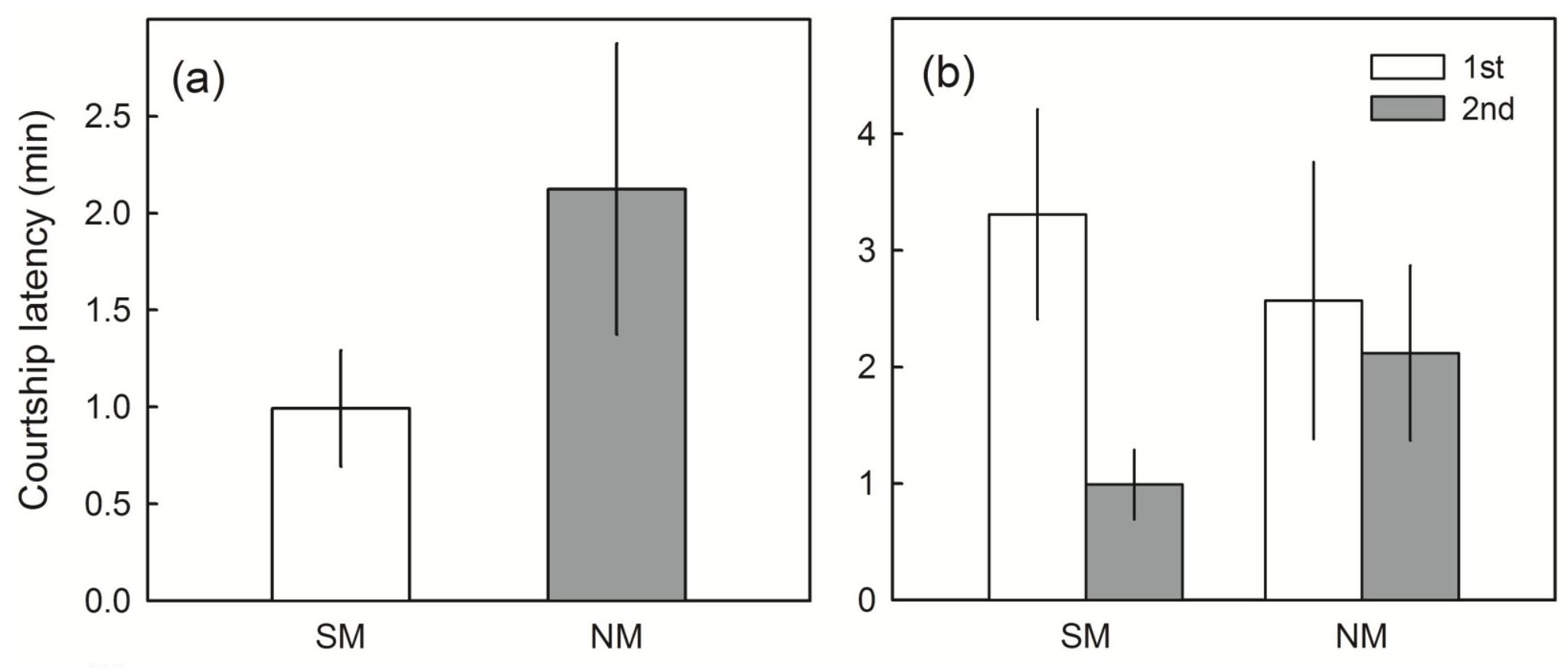

470

471 